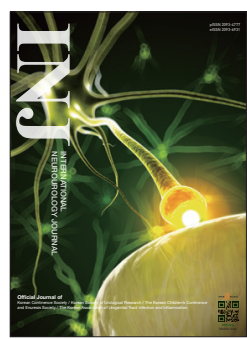

\title{
Time to Say Goodbye to International Neurourology Journal
}

\author{
Hong Sang Moon (iD https://orcid.org/0000-0003-2101-1019 \\ Department of Urology, Hanyang University College of Medicine, Seoul, Korea \\ Email: moonuro@hanyang.ac.kr
}

International Neurourology Journal (INJ)'s 2020 impact factor was calculated as 2.835 , which was the highest score among journals in the field of neurourology. I would like to express my deepest gratitude to the members of INJ's editorial board for their hard work in bringing us to this position. I have been the editor-in-chief of INJ since the September 2019 issue, and before that, I served as an associate editor of INJ. INJ has continued to grow after becoming a SCIE journal in 2014. The people who made INJ a SCIE journal are Professor Tack Lee, the editor-in-chief emeritus, and Professor Khae Hawn Kim. Without their efforts, INJ would not have become a SCIE journal, and it is no exaggeration to say that INJ in its current form has developed thanks to their dedication, hard work, and expertise. Professor Khae Hawn Kim, who will serve as the next editor-inchief of INJ, is also an excellent researcher who is producing outstanding research results in the basic research and clinical fields of neurourology. I have no doubt that Professor Kim will develop INJ even further.

There are many unknown areas in the field of neurourology that have not yet been fully elucidated. Although urologists treat patients as if they have extensive, in-depth knowledge about the physiology, function, and diseases of the organs of the urinary system, in reality, urologists often try to treat diseases without knowing the exact cause or mechanism. An excellent example of this phenomenon is interstitial cystitis/bladder pain syndrome (IC/BPS). The exact cause of IC/BPS is unknown, and there is no definitive treatment for it, so many IC/BPS patients suffer from reduced quality of life and discomfort. Therefore, in my opinion, further research on the mechanisms and treatment of IC/BPS is absolutely necessary. The International Society for the Study of Bladder Pain Syndrome (ESSIC) presents excellent research results on IC/BPS. INJ signed a memorandum of understanding (MOU) with ESSIC last year and became the official journal of ESSIC. I would like to express my gratitude to Professor Lori Birder, the past President Jean Jacques Wyndaele, and President Mauro Cervigni, who have provided vital assistance in creating the MOU between the two organizations.

I would like to thank Professor Karl-Erik Andersson and Professor Lori Birder, who served as INJ's deputy editors-inchief, introduced INJ to researchers in other countries, and submitted excellent articles. I would also like to express my deep gratitude to the associate editors (Professor Khae Hawn Kim, Professor Young Sam Cho, Professor Su Jin Kim) who have spared no effort to help INJ develop, and to Mr. Hwan Tae Ahn, INJ's manuscript editor, who has demonstrated an excellent ability to handle all my requests promptly.

Two years is a period of time that can be short or long to do something. During my 2 years as the editor-in-chief of INJ, I experienced a lot of difficult challenges, and there were many difficult days, but I am fortunate to be able to retire after having achieved good results. I think that it is now time for me to leave because I have done everything I could at INJ. I hope that INJ, with its new editor-in-chief, will become the most brilliant journal in the field of neurourology in the future.

- Conflict of Interest: No potential conflict of interest relevant to this article was reported. 